No. 14 May 2020

\title{
INTEGRAL TRANSPERSONAL JOURNAL
}

of ants, sciences and technologies

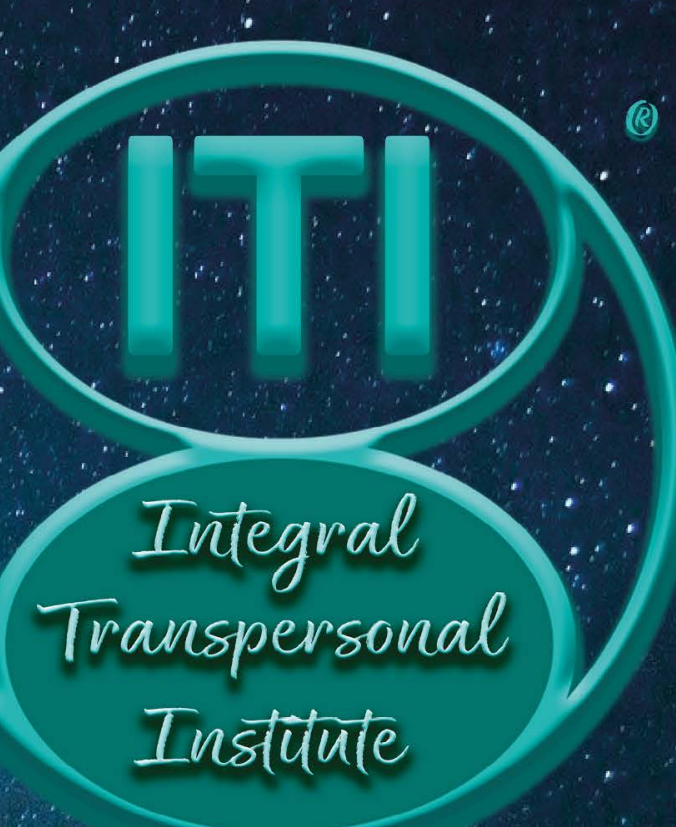

Contributors:

Jevon Dangeli Sven Doeknner Michael Doty Kelly.Kilrea
Stéphanie Larrue Sheena Necole McMahon Magali Ollagnier-Beldame Wendy Smidt 


\section{Integral \\ Transpersonal Journal}

of arts, sciences and technologies

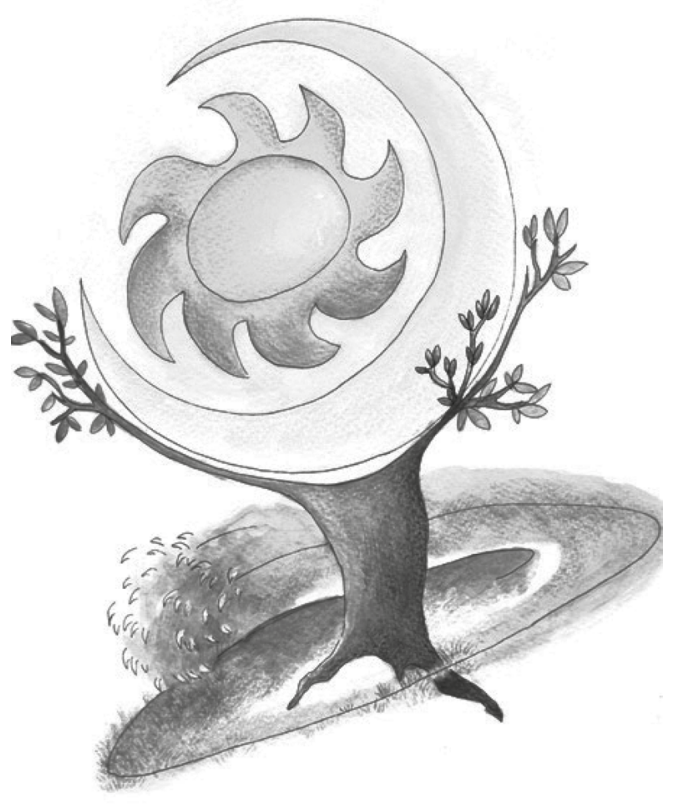




\section{Eurotas Official Journal}

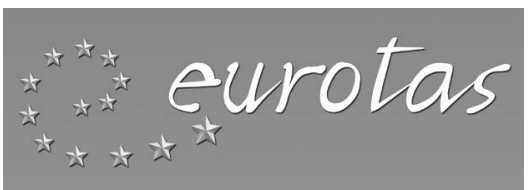




\section{EDITORIAL INFORMATION}

SCIENTIFIC DIRECTOR Pier Luigi Lattuada

EDITORIAL STAFF

SCIENTIFIC BOARD

Jure Biechonsky, Estonia

Bernadette Blin, France

Lyudmila Scortesca, Moldova

Vitor Jose Rodrigues, Portugal

Magda Sole, Spain

Giovanna Calabrese, Italy

Ingrida Indane, Latvia

Vladimir Maykov, Russia

Regina Hess, Germany

Steven Schmitz, USA 


\section{BOARD OF REVIEWERS}

REVIEWERS

TRANSLATORS
Regina U. Hess, Germany - chief

Lindy McMullin, Greece

Rona Newmark, South Africa

Elena Piccoli, Italy

Timoty M. Perazzoli

Valentina D. M. Lattuada 


\section{INTEGRAL TRANSPERSONAL JOURNAL \\ VOLUME XIV, NUMBER XIV, 2020}

\section{Editorial}

A way to.

Tools for a new method of knowing and living

[Rossana Strambaci]

Exploring Experience as a Myriad Richness:

Micro-phenomenology as a

Transformative Approach to Research

(Magali Ollagnier-Beldame)

Engaging the Transformative Potential of Short

Film-making toward Critical Awareness and

Transpersonal Growth amongst Post-school Youth

[Wendy Smidt]

Open Awareness Inquiry. Introducing Applied

Open Awareness as an Inquiry Method

for Researchers to Engage the Transformative

Dimensions of Consciousness

[Jevon Dangeli] 
Virginia Satir's Transformational Systemic Therapy: A

Transpersonal Approach to Family Therapy

[Kelly Kilrea; Stéphanie Larrue]

For a Definition of Gratitude, in Order to Study its

Correlation with Well-being and Self Transcendence

[Sheena Necole McMahon]

Psychedelic Properties of Peganum Harmala:

Macrodose and Microdose Reports

[Michael Doty]

The Listening VOICE:

a Journey of Shamanic Initiation

[Sven Doehner]

Authors' Instructions Text Format

Information about ITI

Notice to subscribers

134 


\section{Editorial}

\section{A way to \\ Tools for a new method of knowing and living}

Rossana Strambaci, Ph.D.

ITJ Editor Director

We can say that many of the main foundations of the philosophy of submissions of this issue of the Journal Edmund Husserl and the concept of relate to "tools", instruments to reach epoché, among other things.

awareness and openness "not only about The "art based inquiry" of Wendy new knowledge, but also about new Smidt refers on a pilot study involving contexts for knowledge and new ways young adults and has the aim to of knowing", as expressed by Magali examine the connection between Ollagner-Beldame, citing Vitor J. creative process and growth of Rodriguez'scontribution ofITJn.0 (p.64). personal and transpersonal awareness. What is at stake here is not only the From a methodological point of content of knowledge as an object, but view, this qualitative data collection also the way to know something. So, requires a targeted and particular the frame of cognitive sciences is the approach, different from the one used critical issue in the work of Ollagner- in quantitative sciences. Furthermore, Beldame on Micro-Phenomenology and most significant, this kind of by an excursus through some of the procedure can originate a form of 
collective consciousness, a sort of "awareness of field".

We are talking about the same collective awareness and creativity that is reached through the way of the Open Awareness Inquiry, to which the contribution of Jevon Dangeli refers. The kind of inquiry treated in Dangeli's study is founded on the idea of an extended and inclusive epistemology. Due to this kind of knowledge process, not only people can reach an "holotropic" state of consciousness, but they can also experience a collective state that opens a "field" of common understanding, disclosure and insight. A kind of cognizance that breathes a common atmosphere, in the same way that contributors and co-researchers breath according to a collective, synchronized rhythm.

The work of Stephanie Larrue and Kelly Kilrea - which is about the Family Therapy of Virginia Satir as a transpersonal therapy applied - gives to us one more tool. Thesystemicapproachtofamilydynamics [characterised by great attention to the whole of family relationships in their interdependencies] in the model of Satir's Transformational Systemic Therapy goes further and expands to a transpersonal perspective. The category of congruence changes, and the concept widens to include not only interpersonal and intrapsychic congruence, but also universal and spiritual congruence.

Then we have the feeling of gratitude, which can be a way to self-transcendence as indicated by Sheena MacMahon's study path. In the work that is presented here, MacMahon gives a review of some studies about measurement scale of gratitude and introduces starting point to future researches and questions on this topic.

The personal account of selfexperimentation with Peganum Harmala, reported by Michael Doty, gives us a glance at what could be an entheogenic applications use of these extracts, as a method to release the transformative potential of human consciousness, an instrument used for millennia in traditional medicine and religious rites.

A very strong medium to "alchemical" transformation, then, is the power of the voice, theme which is developed by Sven Doehner in the report of his personal experience, A Journey of Shamanic initiation. Doehner connects the element of images, as a structuring factor of our experience, with emotions and sounds, arguing that all these elements have the power of create realities. The work explores this powerful 


\section{Editorial}

connection, tracing a method to which the author gave the name of "Sound Imagination".

We although have to say that an instrument is never just a tool, but it is also a choice, a way, a method. It is interesting to point out that the word method has in itself the root of

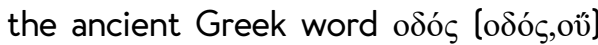
that means way, path. The etymological meaning of the Greek term can be literally translated as "the way to".

$A$ reflection on this theme has been suggested by Pier Luigi Lattuada in a recent lecture with students and colleagues. So, if we stay within a hermeneutical approach to reality meaninganapproach thatrecognisesthat reality is based on the interpretation that of it is given - we get some interesting consequences both on the level of knowledge and on the level of practice. In fact one of the clearest consequences of this mode is the awareness that the method we choose to follow a path defines the way itself and draws the horizon.
If we agree that the Hermeneutics, as a philosophical school of thought, has changed the forma mentis of the Twentieth Century, we are now faced with a further passage regarding the epistemological paradigm.

Reading the research papers presented in this Issue, what catch the eye, in my opinion, is the fact that the horizon drawn by these tools of knowledge is a unitive, circular one, characterised by the sign of sharing.

This implies a two-way dimension between "the knower" and "the known", between "the subject" and "the object" of knowledge, a circular dimension which ceaselessly proposes itself in the context of experience. Even more, this includes the possibility of shared knowledge through non-local connections.

It should not be denied that it was decisive, in past centuries, to acquire a critical consciousness. But now it seems necessary to evolve towards a unitive consciousness, grounded on unitive awareness. 